\title{
Bifurcations of Optimal Solutions for Coordinated Robotic Systems: Numerical and Homotopy Methods
}

\author{
Baoyang Deng, Andrés K. Valenzuela and Bill Goodwine
}

\begin{abstract}
This paper presents the relatively rich and interesting bifurcation structure that is present in the nature of optimal solutions to a multi-robot formation control problem. The problem considered is a two point nonlinear boundaryvalue problem that can only be solved numerically. Since common numerical solution techniques such as the shooting method are local in nature and hence are difficult to use to find multiple solutions, an alternative formulation of the problem is presented that can be solved through homotopy methods for polynomial systems. These methods are guaranteed to find all solutions within the resolution of the system description's discretization. Specifically, this paper studies a group of unicyclelike autonomous mobile robots operating in a 2-dimensional obstacle-free environment. Each robot has a predefined initial state and final state and the problem is to find the optimal path between two states for every robot. The path is optimized with respect to the control effort and the deviation from a desired formation. The bifurcation parameter is the relative weight given to penalizing the deviation from the desired formation versus control effort. It is shown that as this number varies, bifurcations of solutions are obtained. Considering the common use of optimization methods in robotic navigation and coordination problems, understanding the existence and structure of bifurcating and multiple solutions is of great importance in robotics.
\end{abstract}

\section{INTRODUCTION AND BACKGROUND}

Distributed systems with multiple agents have been the focus of many research efforts in recent years. The applications of distributed systems are ubiquitous, including robotic underwater vehicles [1], satellite clustering [2], electric power system [3], search and rescue operations [4] etc. The approaches to the multi-robotic formation control problem are similarly many and varied. Roughly, they can be categorized into three groups: leader-follower methods [5-7], behaviorbased methods [8-10] and virtual structure methods [11-13].

In this paper, the problem addressed is to control a formation of robots moving along an optimal path between an initial configuration and a final configuration. The path is optimized with respect to a combination of the control effort and the deviation from a desired formation. Since each robot has its own predefined initial state and final state, the procedure that standard optimization methods suggest for finding the optimal path is to solve a boundary value for

B. Goodwine is with Faculty of of Aerospace and Mechanical Engineering, University of Notre Dame, Notre Dame, IN 46556, USA jgoodwinend. edu

B. Deng is with the Department of Aerospace and Mechanical Engineering, University of Notre Dame, Notre Dame, IN 46556, USA bdeng2 and. edu

A. Valenzuela is with the Department of Mechanical Engineering at the Massachusetts Institute of Technology, Cambridge, MA 02139, USA
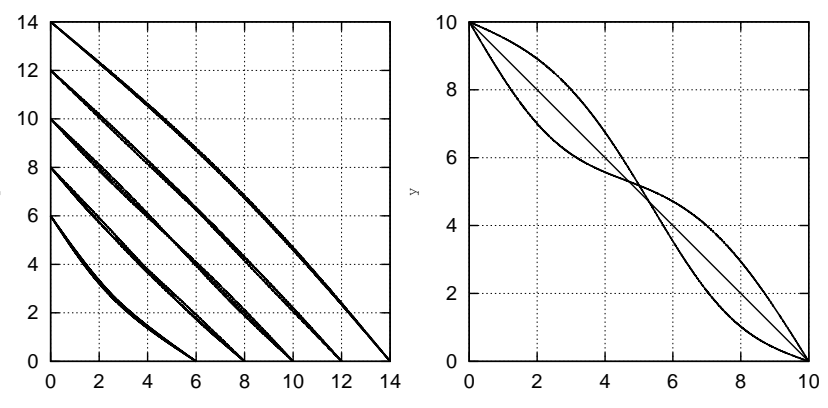

Fig. 1. Optimal paths for the five robot system with $k=24.5$.

a set of second order ordinary differential equations (derived subsequently in Section II).

The existence of multiple nontrivial solutions of BVPs for nonlinear second order ODEs has been investigated by some authors. Not surprisingly, however, the results are not as fully developed as the case for the bifurcation of fixed points for ordinary differential equations. For example, for

$$
\begin{aligned}
x^{\prime \prime}+a(t) f(x) & =0 \\
x(0) & =0 \\
x(1) & =0,
\end{aligned}
$$

the properties of the solutions depend on the limiting behavior of the function $f(u)$. Erbe and Wang [14] studied the existence of positive solutions of the equation with linear boundary conditions. Also, for

$$
\begin{aligned}
f_{0} & =\lim _{s \rightarrow+0} \frac{f(s)}{s} \\
f_{\infty} & =\lim _{s \rightarrow+\infty} \frac{f(s)}{s},
\end{aligned}
$$

they showed the existence of at least one positive solution in two cases, superlinearity $\left(f_{0}=0, f_{\infty}=\infty\right)$ or sublinearity $\left(f_{0}=\infty, f_{\infty}=0\right)$. In [15], Erbe, $\mathrm{Hu}$ and Wang showed that there were at least two positive solutions in the case of superlinearity at one end (zero or infinity) and sublinearity at the other end. Naito and Tanaka [16] and Ma and Thompson [17] established a precise condition concerning the behavior of the ratio $f(s) / s$ for the existence and nonexistence of solutions. Their main result was that the problem had at least $k$ solutions if the ratio $f(s) / s$ crossed the $k$ eigenvalues of the associated eigenvalue problem. For a class of systems of second order ODEs, Marcos do Ó, Lorca and Ubilla [18] 
used the fixed-point theorem of cone expansion/compression type, the upper-lower solutions method and degree arguments to study the existence, nonexistence, and multiplicity of positive solutions of the boundary value problem. While the problems they address are similar in nature to ours, none of these results are, unfortunately, directly applicable to it.

This paper presents bifurcation results for a specific formation control problem. These solutions were found by using the shooting method to solve the nonlinear two-point boundary value problem. The existence of multiple solutions and their bifurcation structure is important for roboticists who deal in motion planning methods that are based on optimization techniques. Knowledge of the existence and nature of bifurcations of solutions of this type are important for practicing engineers because if a solution is found that is optimal, but not necessarily desirable, it may be the case that a different solution for the same cost function exists and is superior. Searching for multiple solutions of an optimization problem is likely to be less costly than reformulating the optimization problem.

Additionally, this paper presents an alternative finitedifference formulation of the problem. When expressed as a finite difference system, an additional transformation allows the boundary value problem to be expressed as a system of algebraic polynomial equations. This is important because methods exists for finding all solutions of polynomial equations, and hence can potentially serve as a means to validate that the solutions found by the search using the shooting method represent all possible solutions. Homotopy continuation methods are based on the simple concept of constructing a system with the same number of roots as the original system where the roots of the new system are known, and then tracing the roots of the known system as its solution is deformed into that of the original system. As general references, see, for example [19-21]. Initial results of the application of this method are presented as first validation that the method is applicable and works. A full exposition will be the subject of a full future publication. Due to the computational cost of the approach, a parallel computing environment and other specialized techniques such as a judicious selection of the homotopy will be necessary.

\section{Problem Statement}

We adopt a simplified version of the kinematic robotic unicycle as a prototypical model. The simple kinematics of this kind of robot are described by

$$
\begin{aligned}
& \dot{x}=u_{1} \\
& \dot{y}=u_{2} .
\end{aligned}
$$

The problem is to find the controls $u_{i_{1}}(t), u_{i_{2}}(t)$ for each robot $i$ that steer a formation of robots of this type from its start configuration to its goal configuration, while maintaining a rigid body formation at the beginning and end of the trajectory and minimizing the global performance index

$$
J=\int_{0}^{t_{f}} \sum_{i=1}^{n}\left(\left(u_{i_{1}}\right)^{2}+\left(u_{i_{2}}\right)^{2}\right)+\sum_{i=1}^{n-1} k\left(d_{i}-\bar{d}\right)^{2} d t
$$

subject to the robotic kinematic constraints in Equation 1, where $n>2$ is the number of robots, $d_{i}=\left(\left(x_{i}-x_{i+1}\right)^{2}+\right.$ $\left.\left(y_{i}-y_{i+1}\right)^{2}\right)^{1 / 2}$ is the Euclidean distance between the $i$ th and $(i+1)$ th robots, $\bar{d}$ is the desired distance between two adjacent robots, and $k$ is a non-negative weighting constant. The cost function minimizes a combination of the control effort (first summation) and the deviation from a desired formation (second summation). The weighting constant, which will serve as our bifurcation parameter, balances maintaining the desired distance between the robots against finding the minimum-cost path based on control effort.

Applying Pontryagin's maximum principle to solve the optimal control problem, we obtain the optimal inputs

$$
\begin{aligned}
u_{i_{1}} & =\frac{1}{2} p_{i_{1}} \\
u_{i_{2}} & =\frac{1}{2} p_{i_{2}}
\end{aligned}
$$

and equations of motion

$$
\begin{aligned}
\dot{x}_{i} & =\frac{1}{2} p_{i_{1}} \\
\dot{y}_{i} & =\frac{1}{2} p_{i_{2}} \\
\dot{p}_{i_{1}} & =\frac{2 k\left(x_{i}-x_{i-1}\right)\left(d_{i-1}-\bar{d}\right)}{d_{i-1}}+\frac{2 k\left(x_{i}-x_{i+1}\right)\left(d_{i}-\bar{d}\right)}{d_{i}} \\
\dot{p}_{i_{2}} & =\frac{2 k\left(y_{i}-y_{i-1}\right)\left(d_{i-1}-\bar{d}\right)}{d_{i-1}}+\frac{2 k\left(y_{i}-y_{i+1}\right)\left(d_{i}-\bar{d}\right)}{d_{i}} .
\end{aligned}
$$

Because they correspond to the robots at the ends of the formation, the last two equations in Equation 2 have only the second term when $i=1$ and only the first term when $i=n$.

The cases considered in this paper are limited to the boundary conditions

$$
\begin{aligned}
& x_{i}(0)=c+(i-1) \bar{d}, \\
& x_{i}(1)=0, \\
& y_{i}(0)=0, \\
& y_{i}(1)=c+(i-1) \bar{d},
\end{aligned}
$$

where $c$ is a constant. These boundary conditions correspond to an initial formation in which the robots are arranged along the $x$-axis with the first robot at at $x=c$ and a distance $\bar{d}$ between each robot and a final formation in which the robots are arranged along the $y$-axis with the first robot at $y=c$ and a distance $\bar{d}$ between each robot, as is shown in the left illustration in Figure 1. It is important to note that if the initial and final formations are not parallel, then straight-line trajectories satisfying the boundary conditions will not, in general, maintain the desired distance between the robots.

\section{BIFURCATION RESUlTS}

For a distributed system containing $n$ robots, when the weighting constant $k$ is given, an optimal trajectory can be obtained numerically by solving the equations of motion given by Equation 2 using the shooting method (see [22]). 


\section{A. Solutions for a five robot system}

The figure on the left in Figure 1 illustrates three different solutions that satisfy the equations of motion in Equation 2 and boundary conditions in Equation 3 for $k=24.5, c=6$ and $\bar{d}=2$ for a formation of five robots. Since the differences among these trajectories are difficult to distinguish on such a small graph, the figure on the right illustrates them for the third (middle) robot with the difference magnified by a factor of 10 .

Since $k$ is a parameter in differential equations, it will clearly affect the solutions. In fact, as $k$ is varied, the nature and number of solutions changes. In our prior work [23] we showed that there is a unique solution to the system in Equation 2 when $k$ is small and in the limit as $k$ approaches infinity, the number of solutions also approaches infinity.

In order to present the relationship between the number of solutions and $k$, we construct a bifurcation diagram as follows: since a straight line connecting end points is the optimal solution when $k=0$, we will designate that as a nominal trajectory. One measure of the difference between solutions would be their deviation from the straight line nominal solution at some specified time. As long as the different solution are not intersecting at that time, this would provide a measure of difference between different solutions. In all the bifurcation diagram illustrated subsequently, $t=$ 0.25 is used. For different formations and different types of robots, a different value of $t$ may be a better choice; however, for all the systems studied in this paper, $t=0.25$ appeared to adequately represent the relationship among the solutions. Also, while alternative measures of differences between the solutions may, in general, be superior, this simple choice appears to suffice for all the cases considered in this paper.

The plots in Figure 2 illustrate this measure of the difference between solutions for each robot in the five robot system as $k$ varies from 0 to 25 . In these bifurcation diagrams, the first robot is the one with the shortest trajectory, the fifth robot is the one with the longest trajectory and they are ordered sequentially. A single branch corresponds to a family of solutions as the bifurcation parameter is varied. If there is more than one branch for a specified $k$ value, then more than one solution was found. Observe that the bifurcation diagrams for robots 1 and 5 are symmetric to each other about $d=0$ axis and the bifurcation diagrams for robots 2 and 4 are similarly symmetric (even though each follows a trajectory with a different length). Finally, the bifurcation diagram for robot 3 is symmetric to itself about $d=0$.

A close analysis of the actual trajectories that the robots follow, illustrated in the figure on the right in Figure 1, reveals that the trajectories themselves are not symmetric (the two trajectories with pronounced curves intersect, but not at a point on the straight line solution). A measure that is based upon the deviation from the nominal solution appears to be necessary to determine the real symmetric nature of the solutions. Reference [23] contains the analysis of the system equations that, when applied to these bifurcation diagrams, proves that these symmetries must, in fact, exist.
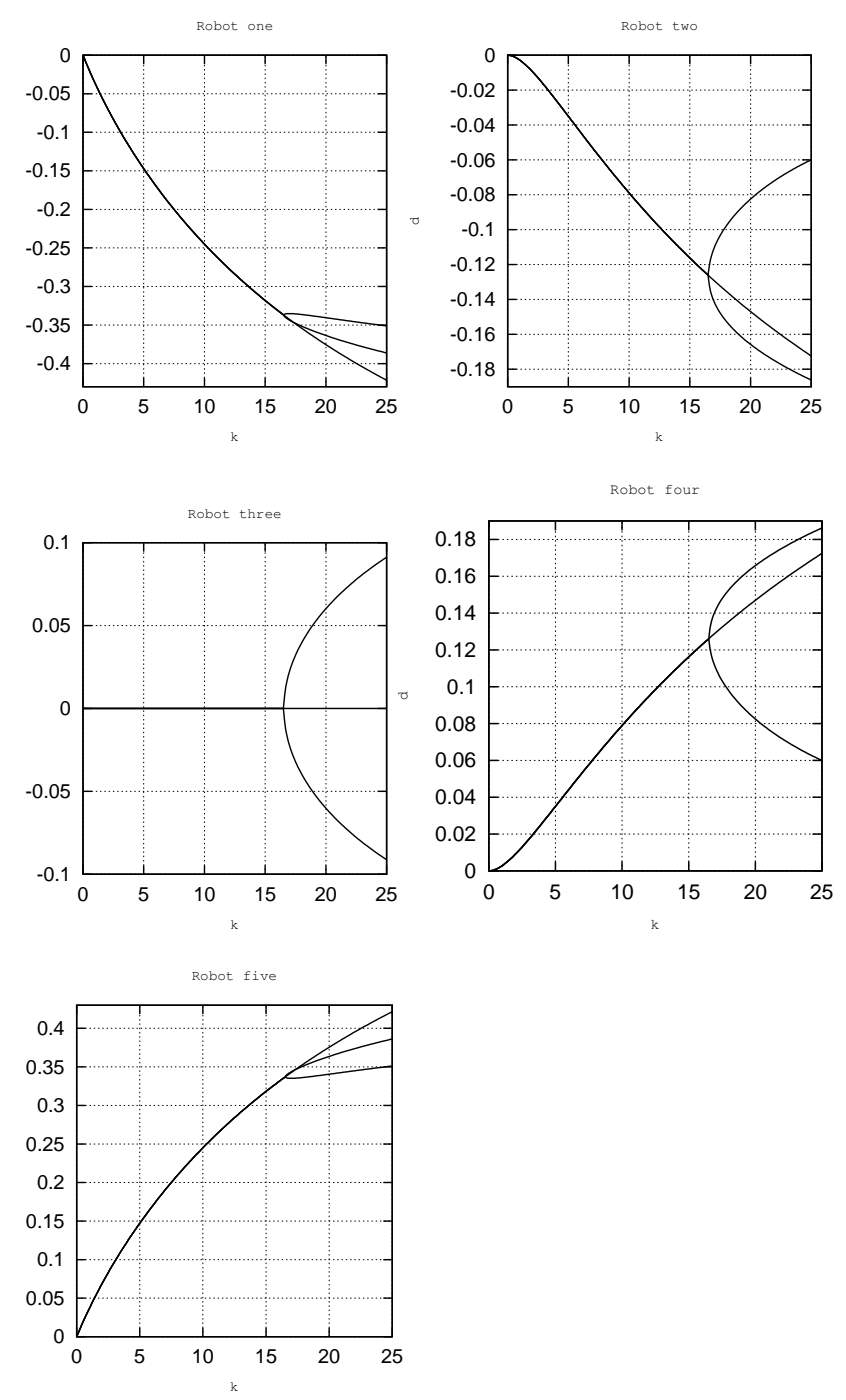

Fig. 2. Bifurcation diagrams for a five robot system.

B. Solutions for a seven robot system

Figures 3 and 4 illustrate similar results for a seven robot system. Figure 3 illustrates the trajectories when $k=24.5$, $c=4$ and $\bar{d}=2$. Again, because the difference between any two trajectories is hard to distinguish in the small left figure, the right figure in Figure 3 illustrates the trajectory with the deviation from the nominal trajectory for the fifth robot magnified by a factor of ten. Figure 4 illustrates the bifurcation diagrams for the solutions versus $k$ constructed in a manner identical to those of the system of five robots. Observe that, similar to their counterparts in the five robot case, the bifurcation diagrams for robots 1 and 7 are symmetric to each other about $d=0$ as was the bifurcation diagrams for robots 2 and 6 and robots 3 and 5, and the bifurcation diagram for robot 4 is symmetric to itself about $d=0$. 


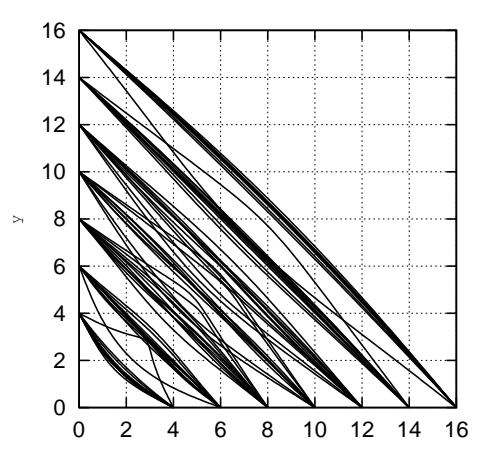

Fig. 3. Optimal paths for a seven robot system with $k=23$

\section{The Finite DiffERENCE Formulation AND HOMOTOPY CONTINUATION}

The equations of motion given in Equation 2 are obviously equivalent to the coupled second order system

$$
\begin{aligned}
& \ddot{x}=\frac{k\left(x_{i}-x_{i-1}\right)\left(d_{i-1}-\bar{d}\right)}{d_{i-1}}+\frac{k\left(x_{i}-x_{i+1}\right)\left(d_{i}-\bar{d}\right)}{d_{i}} \\
& \ddot{y}=\frac{k\left(y_{i}-y_{i-1}\right)\left(d_{i-1}-\bar{d}\right)}{d_{i-1}}+\frac{k\left(y_{i}-y_{i+1}\right)\left(d_{i}-\bar{d}\right)}{d_{i}}
\end{aligned}
$$

where $d_{i}$ is the Euclidean distance between robots $i$ and $i+1$ and $\bar{d}$ is the desired formation distance between them. In the finite difference formulation, the time interval is divided into equal intervals of length $h$, and the values of the state variables at time $t=j h$ are denoted by $x_{i}^{j}, \dot{x}_{i}^{j}, y_{i}^{j}$ and $\dot{y}_{i}^{j}$.

Using the usual finite difference approximation for the second derivative

$$
\ddot{x}_{i}^{j}=\frac{x_{i}^{j+1}-2 x_{i}^{j}+x_{i}^{j-1}}{h^{2}},
$$

the system at time $t=j h$ may be expressed as

$$
\begin{aligned}
\frac{x_{i}^{j+1}-2 x_{i}^{j}+x_{i}^{j-1}}{h^{2}}= & \frac{k\left(x_{i}^{j}-x_{i-1}^{j}\right)\left(d_{j}^{j} i-1-\bar{d}\right)}{d_{i-1}^{j}} \\
& +\frac{k\left(x_{i}^{j}-x_{i+1}^{j}\right)\left(d_{i}^{j}-\bar{d}\right)}{d_{i}^{j}} \\
\frac{y_{i}^{j+1}-2 y_{i}^{j}+y_{i}^{j-1}}{h^{2}}= & \frac{k\left(y_{i}^{j}-y_{i-1}^{j}\right)\left(d_{i-1}^{j}-\bar{d}\right)}{d_{i-1}^{j}} \\
& +\frac{k\left(y_{i}^{j}-y_{i+1}^{j}\right)\left(d_{i}^{j}-\bar{d}\right)}{d_{i}^{j}} .
\end{aligned}
$$

Even if the denominators are cleared, this is not in polynomial form because $d_{i}=\sqrt{\left(x_{i}-x_{i+1}\right)^{2}+\left(y_{i}-y_{i+1}\right)^{2}}$. However, if we treat each $d_{i}^{j}$ as a variable and add the equation $\left(d_{i}^{j}\right)^{2}=\left(x_{i}-x_{i+1}\right)^{2}+\left(y_{i}-y_{i+1}\right)^{2}$, the system is polyno-
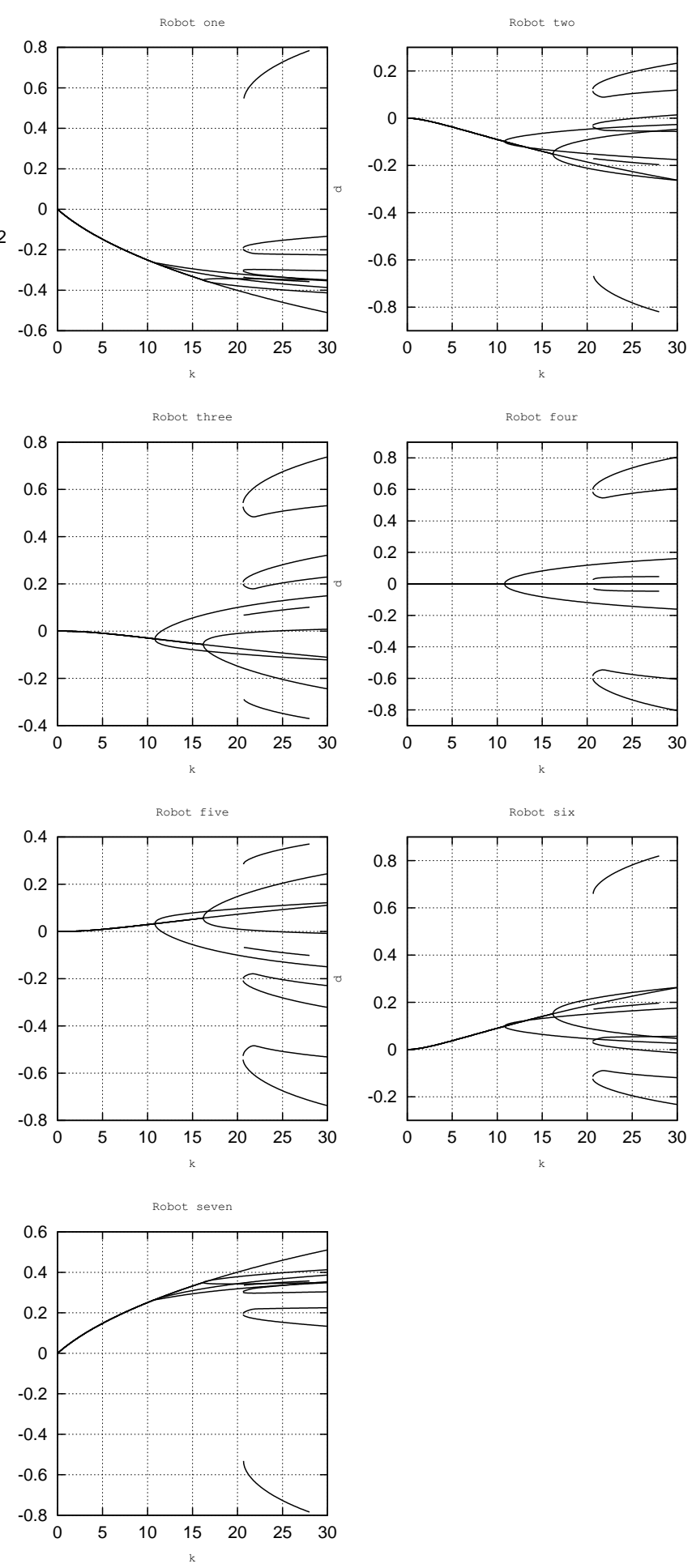

Fig. 4. Bifurcation diagrams for a 7-robotic system 
mial. In particular,

$$
\begin{gathered}
d_{i}^{j} d_{i-1}^{j}\left(x_{i}^{j+1}-2 x_{i}^{j}+x_{i}^{j-1}\right)= \\
h^{2} d_{i}^{j}\left(k\left(x_{i}^{j}-x_{i-1}^{j}\right)\left(d_{j}^{j} i-1-\bar{d}\right)\right) \\
+h^{2} d_{i-1}^{j}\left(k\left(x_{i}^{j}-x_{i+1}^{j}\right)\left(d_{i}^{j}-\bar{d}\right)\right), \\
d_{i}^{j} d_{i-1}^{j}\left(y_{i}^{j+1}-2 y_{i}^{j}+y_{i}^{j-1}\right)= \\
h^{2} d_{i}^{j}\left(k\left(y_{i}^{j}-y_{i-1}^{j}\right)\left(d_{j}^{j} i-1-\bar{d}\right)\right) \\
+h^{2} d_{i-1}^{j}\left(k\left(y_{i}^{j}-y_{i+1}^{j}\right)\left(d_{i}^{j}-\bar{d}\right)\right), \\
\left(d_{i}^{j}\right)^{2}=\left(x_{i}^{j}-x_{i-1}^{j}\right)^{2}+\left(y_{i}^{j}-y_{i-1}^{j}\right)^{2} .
\end{gathered}
$$

Since $x_{i}^{j}, y_{i}^{j}$ and $d_{i}^{j}$ are the variables, the first two equations are third order and the last equation is second order.

In general, the number of roots of polynomial systems scales very poorly. For example, for five robots and two time steps, $i=1, \ldots, 5$ and $j=0,1,2$, there are 15 sets of these equations, and, in general, for 30 third order equations and 15 second order equations there are $3^{30} 2^{15}$ (which is on the order of $10^{18}$ ) roots. However, due to the repeated structure of the equations, the number of actual solutions is greatly reduced. We utilize a solver called Bertini [24], which uses homotopy continuation methods to find the roots of the polynomial system and also is capable of preprocessing the system to reduce the possible number of solutions based on system symmetries, etc.

Using this approach and the Bertini software on the five robot system with two time steps, $j=0,1,2$ for several small values of $k$ validates the unique solutions in the bifurcation diagrams (the initial isolated branch in Figures 2 and 4). As a representative case, for $k=2.0$, Bertini found 68 total solutions, 16 of which were real. Because the distance between robots is a state, only solutions for which every distance is positive is a feasible solution, and of the 16 real solutions, only one had positive values for all of the distance variables. For various values of $k$ between zero and 2.5 , the polynomial solutions tracked those illustrated in the bifurcation diagrams. Running on a $2.4 \mathrm{GHz}$ Intel Core 2 Duo Macbook running Mac OS 10.5.8, it took approximately one hour to determine all of the solutions.

Extending these results to validate and reconstruct the entire bifurcation diagrams is the focus of our current efforts. Bertini scales well in a parallel computing environment because of the nature of homotopy tracking solutions from one polynomial to another. Furthermore, it allows a "user defined homotopy" from which we can use the known solution for one $k$ value as the starting solution for nearby $k$ values. This will allow us to reconstruct the bifurcation diagrams using a method that is guaranteed to find all solutions. Hence, this approach will find branches that were perhaps simply not found in our search using the shooting method or solutions that are not numerically stable using the shooting method.

\section{CONClusions AND Future Work}

This paper considers the optimal control problem for a formation of multiple robots. The trajectory of each robot is optimized with respect to a combination of the control effort and the deviation from a desired formation, which in this paper is simply a formation that maintains a specified distance between adjacent robots. The paper first presents numerical results illustrating the structure of bifurcations and multiple solutions of the second order nonlinear boundary value problem associated with the optimal control problem.

Initial results which validate the approach using the finite difference formulation which may be transformed into a polynomial system were also presented. They are consistent with the results using the shooting method for several small values of the bifurcation parameter. Due to the complexity of applying this approach to reconstruct the entire bifurcation diagram, that work will be the subject of a future presentation

An additional focus of future efforts relates to generalizing the results. The results presented in this paper that are specific to the system studied are likely to be much more general than the particular case presented in this paper. Determining the most general classes of robots and formations that maintain the symmetry properties of the results and similar bifurcation structure is of interest.

\section{ACKNOWLEDGMENTS}

The authors gratefully acknowledge the very useful discussions with Andrew Sommese.

\section{REFERENCES}

[1] T. R. Smith, H. Hanssmann, and N. E. Leonard. Orientation control of multiple underwater vehicles with symmetry-breaking potentials. In 40th IEEE Conference on Decision and Control, pages 4598-4603, 2001.

[2] C. R. McInnes. Autonomous ring formation for a planar constellation of satellites. AIAA Journal of Guidance Control and Dynamics, 18(5):1215-1217, 1995.

[3] H. Puttgen, P. MacGrego, and F. Lambert. Distributed generation: semantic hype or dawn of a new era. IEEE Power and Energy Magazine, 1:22-29, 2003.

[4] J. Jennings, G. Whelan, and W. Evans. Cooperative search and rescue with a team of mobile robots. In IEEE International Conference on Advanced Robotics, pages 193-200, 1997.

[5] Jaydev P. Desai. Modeling multiple teams of mobile robots: a graph theoretic approach. In Proceedings of the 2001 IEEE/RSJ International Conference on Intelligent Robots ans Systems, pages 381-386, 2001.

[6] Jaydev P. Desai, Jim Ostrowski, and Vijiay Kumar. Controlling formation of multiple mobile robots. In IEEE International Conference on Robotics and Automation, pages 16-21, 1998.

[7] N. E. Leonard and E. Fiorelli. Virtual leaders, artificial potentials, and coordinated control of groups. In 40th IEEE Conference on Decision and Control, pages 2968-2973, 2001. 
[8] T. Balch and R. Arkin. Behavior-based formation control for muti-robotic teams. IEEE Transactions on Robotics and Automation, 14(6):926-934, 1998.

[9] T. Balch and M. Hybinette. Behavior-based coordination of large-scale robot formations. In Fourth International Conference on MultiAgent Systems, 2000.

[10] Zhibao Su and Jilian Lu. Formation feedback applied to behavior-based approach to formation keeping. Jounal of Beijing Institute of Technology, 13(2):190-193, 2004.

[11] Calin Belta and Vijay Kumar. Geometric Methods for Multi-Robot optimal Motion Planning, Handbook of Computational Geometry for Pattern Recognition, Computer Vision, Neurocomputing and Robotics. Springer-Verlag, Berlin, 2005.

[12] M. A. Lewis and K. H. Tan. High precision formation control of mobile robots using virtual structures. Autonomous Robots, 4:387-403, 1997.

[13] Kar-Han Tan and M. Anthony Lewis. Virtual structures for high-precision cooperative mobile robotic control. International Conference on Intelligent Robots and Systems, pages 132-139, 1996.

[14] L.H. Erbe and H. Wang. On the existence of positive solutions of ordinary differential equations. In preceedings of the American Mathematical Society, pages 743748, 1994.

[15] L. Erbe, S. Hu, and H. Wang. Multiple positive solutions of some boundary value problems. Mathematical Analysis and Applications, 184:743-748, 1994.

[16] Y. Naito and S. Tanaka. On the existence of multiple solutions of the boundary value problem for nonlinear second-order differential equations. Nonlinear Analysis, 56(4):919-935, 2004.
[17] Ruyun Ma and Bevan Thompson. Multiplicity results for second-order two-point boundary value problems with nonlinearities across several eigenvalues. Applied Mathematics Letters, 18(5):587-595, 2005.

[18] J. Marcos do Ó, S. Lorca, and P. Ubilla. Local superlinearity for elliptic systems involving parameters. Journal of Differential Equations, 211(1):1-19, 2005.

[19] T.Y. Li. Numerical solution of multivariate polynomial systems by homotopy continuation methods. Acta Numerica, 6:399-436, 1997.

[20] A. Sommese, J. Verschelde, and C. Wampler. Introduction to numerical algebraic geometry. In A. Dickenstein and I. Z. Emiris, editors, Algorithms and Computation in Mathematics, pages 339-392.

[21] A. Sommese, J. Verschelde, and C. Wampler. Solving polynomial systems equation by equation. In A. Dickenstein, F. Schreyer, and A. Sommese, editors, Algorithms in Algebraic Geometry, volume 146 of The IMA volumes in mathematics and its applications, pages 133-152. Springer, 2008.

[22] J. Stoer and R. Bulirsch. Introduction to Numerical Analysis. Springer-Verlag, New York, 1980.

[23] Baoyang Deng, Mihir Sen, and Bill Goodwine. Bifurcations and symmetries of optimal solutions for distributed robotic systems. In Proceedings of the 2009 American Control Conference, 2009.

[24] Daniel J. Bates, Jonathan D. Hauenstein, Andrew J. Sommese, and Charles W. Wampler. http://www. nd.edu/ sommese/bertini/. 